\title{
AMENABLE GROUP ACTIONS ON THE INTEGERS; AN INDEPENDENCE RESULT
}

\author{
MATTHEW FOREMAN
}

Issues involving the uniqueness of Lebesgue measure led to questions as to what extent a group $G$ acting on a set $X$ determines the structure (and number) of $G$-invariant finitely additive probability measures on $X(G$ invariant means). For example, Banach [B] showed that there was more than one rotation invariant finitely additive probability measure on the measurable subsets of $S^{1}$. More recently Sullivan[S] and independently Margulis [M1, M2] (for $n \geq 4$ ) and Drinfield [D] (for $n=2,3$ ) showed that Lebesgue measure is the unique finitely additive rotation invariant measure on the Lebesgue measurable subsets of $S^{n}$. An easier example is the following: Let $\mu$ be any two-valued finitely additive probability measure defined on all subsets of the natural numbers. Let $G$ be the group of all permutations of $\mathbf{N}$ that are equal to the identity function on a set of $\mu$-measure one. Then $\mu$ is the unique $G$-invariant finitely additive probability measure on $\mathscr{P}(\mathbf{N})$. Rosenblatt, noting that all of the known instances of uniqueness involved nonamenable groups, asked whether an amenable group $G$ acting on a set $X$ could uniquely determine a finitely additive invariant probability measure on $X$. (For amenable groups invariant measures always exist.) The main result of this note (Corollary to Theorem 3 ) is that in the concrete case of locally finite (hence amenable) groups acting on the natural numbers, the question of whether there is a $G$ with a unique invariant mean is independent of the standard axioms for mathematics (Zermelo-Fraenkel set theory with the axiom of choice; ZFC).

We begin with some negative results. Let $G$ act on a set $X$. Rosenblatt and Talagrand [RT] showed that if $G$ is nilpotent, then $G$ does not determine a unique invariant mean on $X$. Krasa [K] improved this to solvable groups.

Let $\mathbf{N}$ ! be the group of permutations of the natural numbers with the topology of pointwise convergence. Recall that an analytic subset $A$ of $\mathbf{N}$ ! is a projection of a Borel set $B \subseteq \mathbf{N} ! \times \mathbf{N}$ ! onto the first coordinate. In particular any Borel set is analytic.

TheOREM 1. If $G \subseteq \mathbf{N}$ ! is an analytic amenable group then $G$ does not determine a unique invariant mean on $\mathscr{P}(\mathbf{N})$.

COROLlARY. No countable group determines a unique invariant mean.

The idea of the proof is to show that if $G$ has a unique invariant mean $\mu$, then this mean is determined by $G$ in a very concrete (positive $\Sigma_{1}^{1}(G)$ )

Received by the editors February 1, 1989 and, in revised form, May 17, 1989.

1980 Mathematics Subject Classification (1985 Revision). Primary 28D15, 60B99; Secondary $43 \mathrm{~A} 07$. 
way. If $G$ had the property of Baire, then so would $\mu$, but no nonatomic finitely additive probability measure can have the property of Baire. As a corollary of the proof, one sees that Large Cardinal axioms imply that there is no projective subgroup of $\mathbf{N}$ ! with a unique invariant mean (although there is such a P.C.A. group in L).

The relationship between Theorem 1 and the results involving the algebraic structure of groups is not known.

Yang [Y] showed that under the Continuum Hypothesis there is a locally finite group of permutations of $\mathbf{N}$ admitting a unique invariant mean $\mu: \mathscr{P}(\mathbf{N}) \rightarrow[0,1]$. Yang's mean is surjective. Yang asked whether the result followed from Martin's Axiom.

TheORem 2. Assume Martin's Axiom and let $\mu$ be any two-valued, nonatomic finitely additive probability measure defined on $\mathscr{P}(\mathbf{N})$. Then there is a locally finite group $G$ of permutations of $\mathbf{N}$ having $\mu$ as its unique invariant mean.

In the proof we use the following well-known fact.

FACT. Assume Martin's Axiom. Let $U$ be a nonprincipal ultrafilter on $\mathbf{N}$ and enumerate $\mathscr{P}(\mathbf{N}) \backslash U=\left\langle Y_{\alpha}: \alpha \in c\right\rangle$. Then there is an almost disjoint sequence $\left\langle A_{\alpha}: \alpha \in c\right\rangle \subseteq \sim U$ such that

(1) $A_{\alpha} \cap Y_{\beta}$ is finite for $\beta<\alpha$,

(2) $A_{\alpha} \cap Y_{\alpha}=\varnothing$.

Sketch of the proof of Theorem 2. Let $\mu$ be a two-valued finitely additive probability measure on $\mathscr{P}(\mathbf{N})$. Then $\{X: \mu(X)=1\}$ is an ultrafilter. Let $\left\langle Y_{\alpha}: \alpha \in c\right\rangle=\mathscr{P}(\mathbf{N}) \backslash U$ and let $\left\langle A_{\alpha}: \alpha \in c\right\rangle$ be as in the fact. (This is the only use of Martin's Axiom.)

Let $\left\langle A_{\alpha}^{n}: n \in \mathbf{N}\right\rangle$ be a partition of $A_{\alpha}$ into infinite disjoint sets. Choose bijections $s_{\alpha}^{n}: A_{\alpha}^{n} \rightarrow A_{\alpha}^{n+1}$ and $t_{\alpha}: Y_{\alpha} \rightarrow A_{\alpha}^{0}$. Our group $G$ will be generated by $\left\{\sigma_{\alpha}^{n}: n \in \mathbf{N}, \alpha<c\right\} \cup\left\{\tau_{\alpha}: \alpha<c\right\}$ where

$\sigma_{\alpha}^{n}(k)=\left\{\begin{array}{ll}k, & k \notin A_{\alpha}^{n} \cup A_{\alpha}^{n+1}, \\ s_{\alpha}^{n}(k), & k \in A_{\alpha}^{n}, \\ \left(s_{\alpha}^{n}\right)^{-1}(k), & k \in A_{\alpha}^{n+1},\end{array} \tau_{\alpha}(k)= \begin{cases}k, & k \notin Y_{\alpha} \cup A_{\alpha}^{0}, \\ t_{\alpha}(k), & k \in Y_{\alpha}, \\ t_{\alpha}^{-1}(k), & k \in A_{\alpha}^{0} .\end{cases}\right.$

Note that every element of $G$ is equal to the identity on a set of $\mu$ measure one, hence $\mu$ is $G$-invariant. Suppose $\mu(Y)=0$. Then $Y=Y_{\alpha}$ some $\alpha$. But $Y_{\alpha}$ is $G$-isomorphic to $A_{\alpha}^{n}$ for all $n$. Hence $Y$ has measure zero for all $G$-invariant probability measures, so $\mu$ is the unique $G$-invariant probability measure.

It remains to show that for all $\alpha_{1}, \ldots, \alpha_{k}$ and all $N,\left\{\sigma_{\alpha_{i}}^{n}: i<k, n \leq\right.$ $N\} \cup\left\{\tau_{\alpha_{i}}: i<k\right\}$ generates a finite group $H$. The proof of this shows by induction on $\alpha_{k}$ that there is a $B \in \mathbf{N}$ such that for all $m \in \mathbf{N}$, $|H m| \leq B$.

Two remarks are appropriate. First, the construction shows that under Martin's Axiom, for any $n \in \mathbf{N}$ there is a locally finite group $G$ such that the collection of $G$-invariant means has dimension $n$. Secondly, the construction is only as complicated as a well ordering of the reals. So in $\mathbf{L}$ there is a $\Sigma_{2}^{1}$ group with a unique invariant mean. 
R. McKenzie [M] showed that every locally finite group can be embedded in a locally finite group of the same cardinality with no subgroup of countable index. As a consequence, assuming Martin's Axiom, there are locally finite groups $G<H$ such that there is a $G$-action on $\mathbf{N}$ admitting a unique invariant mean, but every $H$-action on $\mathbf{N}$ admits many invariant means. The author conjectures that under Martin's Axiom there is a locally finite group $G$ and two injections $\varphi_{1}, \varphi_{2}: G \rightarrow \mathbf{N}$ ! such that $\varphi_{1}$ “ $G$ admits a unique invariant mean but $\varphi_{2}$ " $G$ admits many (and both actions are transitive). This would show that "admitting a unique invariant mean" is not an algebraic property.

In the following $M$ will denote a model of the axioms of mathematics and $M[\mathscr{F}]$ will denote another model extending $M$, constructed by the method of forcing [Ku].

THEOREM 3. Let $M \models Z F C+C . H$. Let $\mathscr{F}$ be $M$-generic for the partial ordering adding $\aleph_{2}$-Cohen reals. Then $M[\mathscr{F}] \vDash$ "every locally finite group of permutations of $\mathbf{N}$ has at least two invariant means."

COROLlaRY. The following statement is independent of ZFC,

(*) There is a locally finite group of permutations of $\mathbf{N}$ with a unique invariant finitely additive probability measure.

Proof of Corollary. Theorem 2 (or Yang's theorem) shows $(*)$ is consistent with ZFC. Theorem 3 shows that it is consistent with ZFC that $(*)$ is false.

SKETCH OF PROOF OF TheOREM 3. Towards a contradiction, let $G \in$ $M[\mathscr{F}]$ be a locally finite group with a unique invariant mean $\mu$, where $\mathscr{F}=\left\langle C_{\alpha}: \alpha<\omega_{2}\right\rangle$. We view each Cohen real $C_{\alpha}$ as a subset of $\mathbf{N}$. We may assume that $\aleph_{2}$ of the Cohen reals have $\mu\left(C_{\alpha}\right)<1$, otherwise we replace each real by its complement. Standard amenability considerations show that for each Cohen real $C_{\alpha}$ with $\mu\left(C_{\alpha}\right)<1$ there is a finite subgroup $H_{\alpha} \subseteq G$ such that for all $n \in \mathbf{N}, H_{\alpha} \cdot n \nsubseteq C_{\alpha}$. Let $\tau_{\alpha}$ be a term for $H_{\alpha}$.

Usual arguments show that for a set $S \subseteq \aleph_{2}$ of size $\aleph_{2}$,

(1) The supports of $\left\{\tau_{\alpha}: \alpha \in S\right\}$ for a $\Delta$-system with kernel $K$.

(2) $M\left[\left\langle C_{\alpha}: \alpha \in K\right\rangle\right] \vDash$ C.H. so we may assume $K=\varnothing$ and the supports are disjoint.

(3) Each of the supports, $\rho_{\alpha}$, has the same order type and for all $\alpha, \beta \in S$, $\tau_{\alpha} \mid \rho_{\alpha}$ is isomorphic to $\tau_{\beta} \mid \rho_{\beta}$ (in the obvious sense).

By taking a bijection $b: \omega \rightarrow$ o.t. $\left(\operatorname{supp} \tau_{\beta}\right)$ we show:

There is a term $\tau$ in $M^{\mathbf{P}}$, where $\mathbf{P}$ is the partial ordering for adding $\omega$ Cohen reals $\left\langle x_{n}: n \in \omega\right\rangle$ such that: if $\mathscr{F}_{1} \times \mathscr{F}_{2} \subseteq \mathbf{P} \times \mathbf{P}$ is $M$-generic, then

(a) $\tau^{M\left[\mathscr{F}_{i}\right]}$ is a finite group of permutations of $\mathbf{N}$,

(b) for all $m \in \mathbf{N}, x_{0} \nsupseteq \tau^{M\left[\mathscr{R}_{i}\right.} m$,

(c) $\left\langle\tau^{M\left[\mathscr{F}_{1}\right]}, \tau^{M\left[\mathscr{F}_{2}\right]}\right\rangle \subseteq \mathbf{N}$ ! is finite.

We claim that this yields a contradiction. Let $(p, q) \in \mathbf{P} \times \mathbf{P}$,

$$
(p, q) \vDash\left|\left\langle\tau^{M\left[\mathscr{F}_{1}\right]}, \tau^{M\left[\mathscr{F}_{2}\right]}\right\rangle\right| \leq B .
$$

Using (b) one proves: 
SubClaim. If $p^{\prime} \leq p$ and $\operatorname{supp} p^{\prime} \subseteq N \times N$ and if $X \subseteq \mathbf{N},|X| \geq B N+1$ there is an $m \in X$, such that $\left\{k:\left\|k \in \tau^{M\left[\mathscr{F}_{1}\right]} m\right\| \wedge p^{\prime} \neq 0\right\}$ is infinite (and similarly for $q^{\prime} \leq q$ ).

Applying the subclaim, one builds a sequence $k_{0}, k_{1}, \ldots, k_{B+1} \in \mathbf{N}$ and a descending sequence $\left\langle\left(p_{i}, q_{i}\right): i \leq B+1\right\rangle \subseteq \mathbf{P} \times \mathbf{P}$ with $\left(p_{0}, q_{0}\right)=(p, q)$ such that $\left(p_{2 i}, q_{2 i}\right) \Vdash k_{2 i+1} \in \tau^{M\left[\mathscr{F}_{1}\right]} k_{i}$ and $\left(p_{2 i+1}, q_{2 i+1}\right) \Vdash k_{2 i+2} \in \tau^{M\left[\mathscr{F}_{2}\right]} k_{2 i+1}$. This implies that $\left(p_{B+1}, q_{B+1}\right) \Vdash$ "the $\left\langle\tau^{M\left[\mathscr{F}_{1}\right]}, \tau^{N\left[\mathscr{F}_{2}\right]}\right\rangle$-orbit of $k_{0}$ has size $>B$." This contradicts the choice of $(p, q)$.

One can also find other information about groups with invariant means, in particular:

THEOREM 4. (a) Martin's Axiom implies that every locally finite group of cardinality $<c$ has $2^{2^{c}}$ invariant means.

(b) In the model $M\left[\aleph_{2}\right.$ iterated Sacks reals ] [B-L] (where $M \models Z F C+$ C.H.), there is a locally finite group of cardinality $\aleph_{1}$ with a unique invariant mean. Note that in this model $\aleph_{1}<c$.

So,while it is not possible for a countable amenable group to determine a unique invariant means, it is consistent and independent that there exists an amenable group of cardinality $<c$ which has a unique invariant mean.

\section{BIBLIOGRAPHY}

[B] S. Banach, Sur le problème de la mesure, Fund. Math. 4 (1923), 7-33.

[B-L] J. Baumgartner and R. Laver, Iterated perfect set forcing, Annals of Math. Logic 17 (1979), 271-288.

[C] C. Chou, The exact cardinality of the set of invariant means on a group, Proc. Amer. Math. Soc. (1976), 103-106.

[D] V. G. Drinfield, Solution of the Banach-Ruziewicz problem on $S^{2}$ and $S^{3}$, Functional Anal. Appl. 18 (1984), 77-78.

[K] S. Krasa, Non-uniqueness of invariant means for amenable group actions, Monatsh. Math. 100 (1985), 121-125.

[Ku] K. Kunen, Set Theory: an introduction to independence proofs, Elsevier, NorthHolland, 1980, New York.

[M] R. Mckenzie, private correspondence. 235.

[M1] G. Margulis, Some remarks on invariant means, Monatsh. Math. 90 (1980), 233-

[M2] Finitely additive invariant measures on Euclidean spaces, J. Ergodic Theory and Dynamical Systems 2 no. 3 (1982), 383-396.

[R-T] J. Rosenblatt and M. Talagrand, Differential types of invariant means, J. London Math. Soc. 24 (1981), 525-532.

[S] D. Sullivan, For $n>3$, there is only one finitely additive rotationally invariant measure on the n-sphere defined on all Lebesgue measurable sets, Bull. Amer. Math. Soc. (N.S.) 4 (1981), 121-123.

[Y] Z. Yang, Action of amenable groups and uniqueness of invariant means (to appear).

Department of Mathematics, Ohio State University, Columbus, Ohio 43210 